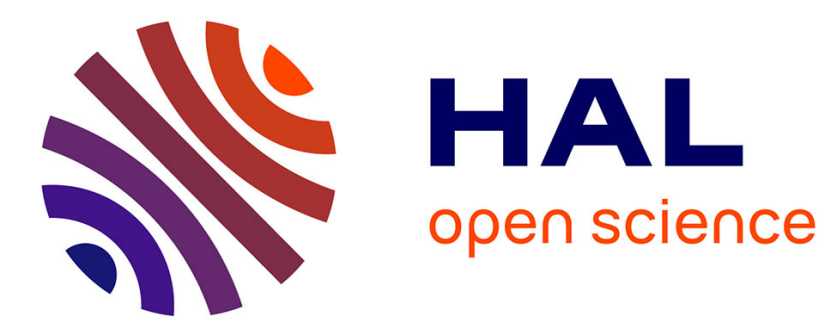

\title{
GCNBoost: Artwork Classification by Label Propagation through a Knowledge Graph
}

Cheikh Brahim El Vaigh, Noa Garcia, Benjamin Renoust, Chenhui Chu, Yuta Nakashima, Hajime Nagahara

\section{- To cite this version:}

Cheikh Brahim El Vaigh, Noa Garcia, Benjamin Renoust, Chenhui Chu, Yuta Nakashima, et al.. GCNBoost: Artwork Classification by Label Propagation through a Knowledge Graph. ICMR 2021 ACM International Conference on Multimedia Retrieval, Aug 2021, Taipei, Taiwan. hal-03228787

\section{HAL Id: hal-03228787 \\ https://hal.inria.fr/hal-03228787}

Submitted on 18 May 2021

HAL is a multi-disciplinary open access archive for the deposit and dissemination of scientific research documents, whether they are published or not. The documents may come from teaching and research institutions in France or abroad, or from public or private research centers.
L'archive ouverte pluridisciplinaire HAL, est destinée au dépôt et à la diffusion de documents scientifiques de niveau recherche, publiés ou non, émanant des établissements d'enseignement et de recherche français ou étrangers, des laboratoires publics ou privés. 


\section{GCNBoost: Artwork Classification by Label Propagation through a Knowledge Graph}

\author{
Cheikh Brahim El Vaigh \\ cheikh-brahim.el-vaigh@irisa.fr \\ Univ. Rennes, CNRS, IRISA \\ Lannion, France
}

\author{
Chenhui Chu \\ chu@ids.osaka-u.ac.jp \\ Kyoto University, and Osaka \\ University, Institute for Datability \\ Science \\ Osaka, Japan
}

\author{
Noa Garcia \\ noagarcia@ids.osaka-u.ac.jp \\ Osaka University, Institute for \\ Datability Science \\ Osaka, Japan
}

\author{
Yuta Nakashima \\ n-yuta@ids.osaka-u.ac.jp \\ Osaka University, Institute for \\ Datability Science \\ Osaka, Japan
}

\author{
Benjamin Renoust \\ renoust@ids.osaka-u.ac.jp \\ Median Technologies, and Osaka \\ University, Institute for Datability \\ Science \\ Valbonne, France
}

\author{
Hajime Nagahara \\ nagahara@ids.osaka-u.ac.jp \\ Osaka University, Institute for \\ Datability Science \\ Osaka, Japan
}

\begin{abstract}
The rise of digitization of cultural documents offers large-scale contents, opening the road for development of AI systems in order to preserve, search, and deliver cultural heritage. To organize such cultural content also means to classify them, a task that is very familiar to modern computer science. Contextual information is often the key to structure such real world data, and we propose to use it in form of a knowledge graph. Such a knowledge graph, combined with content analysis, enhances the notion of proximity between artworks so it improves the performances in classification tasks. In this paper, we propose a novel use of a knowledge graph, that is constructed on annotated data and pseudo-labeled data. With label propagation, we boost artwork classification by training a model using a graph convolutional network, relying on the relationships between entities of the knowledge graph. Following a transductive learning framework, our experiments show that relying on a knowledge graph modeling the relations between labeled data and unlabeled data allows to achieve state-of-the-art results on multiple classification tasks on a dataset of paintings, and on a dataset of Buddha statues. Additionally, we show state-of-the-art results for the difficult case of dealing with unbalanced data, with the limitation of disregarding classes with extremely low degrees in the knowledge graph.
\end{abstract}

\section{CCS Concepts}

- Computing methodologies $\rightarrow$ Image representations; $\bullet$ Applied computing $\rightarrow$ Fine arts.

\section{Keywords}

GCN, Artwork classification, Knowledge graph, label propagation

ICMR '21, August 21-24, 2021, Taipei, Taiwan

(C) 2021

ACM ISBN 978-1-4503-8463-6/21/08 .. \$15.00

https://doi.org/10.1145/3460426.3463636
ACM Reference Format:

Cheikh Brahim El Vaigh, Noa Garcia, Benjamin Renoust, Chenhui Chu, Yuta Nakashima, and Hajime Nagahara. 2021. GCNBoost: Artwork Classification by Label Propagation through a Knowledge Graph. In Proceedings of the 2021 International Conference on Multimedia Retrieval (ICMR '21), August 21-24, 2021, Taipei, Taiwan. ACM, New York, NY, USA, 9 pages. https://doi. org $/ 10.1145 / 3460426.3463636$

\section{Introduction}

Knowledge graphs (KGs), often used for content representation and retrieval, are powerful tools for multimedia data management e.g. $[2,9,38]$. They allow to model data as a set of entities (nodes) and the relations between them (edges). KGs can play a key role to support automatic systems developed to help preserving cultural heritage [1], such as classifying and retrieving historical newspapers [9], paintings [11], or Buddha statues [31]. Automatic artwork classification consists in classifying artworks according to attributes e.g. style, author, or time period [24, 26, 36].

A piece of art, beyond its visual aspect, bears a lot of contextual information (e.g. time, author), which plays an important role in defining the artwork itself (this is especially true for contemporary art that has narrower context than the classical art). Combining visual and contextual information has been shown to be a successful approach for artwork classification [11]. In [11], the contextual information is gathered in a KG to model the interactions between artworks and their attributes, which includes a semantic proximity that might not reside in the visual information itself. In our context, we combine the visual features of a given piece of art with its information embedded in an extended knowledge graph (EKG) that we define hereafter, with different digital archives.

Existing approaches for artwork classification are based on inductive learning [27] generalizing tons of observation, but limited by the cost of laborious human annotation. In contrast, transductive learning and label propagation $[15,44]$ can be used to learn from a smaller set and missing labels. Label propagation can predict pseudo-labels for unlabeled data (test data) and increase the amount of training samples at training time. KGs promote this transductive process $[10,15,33]$ by modeling latent relations between 


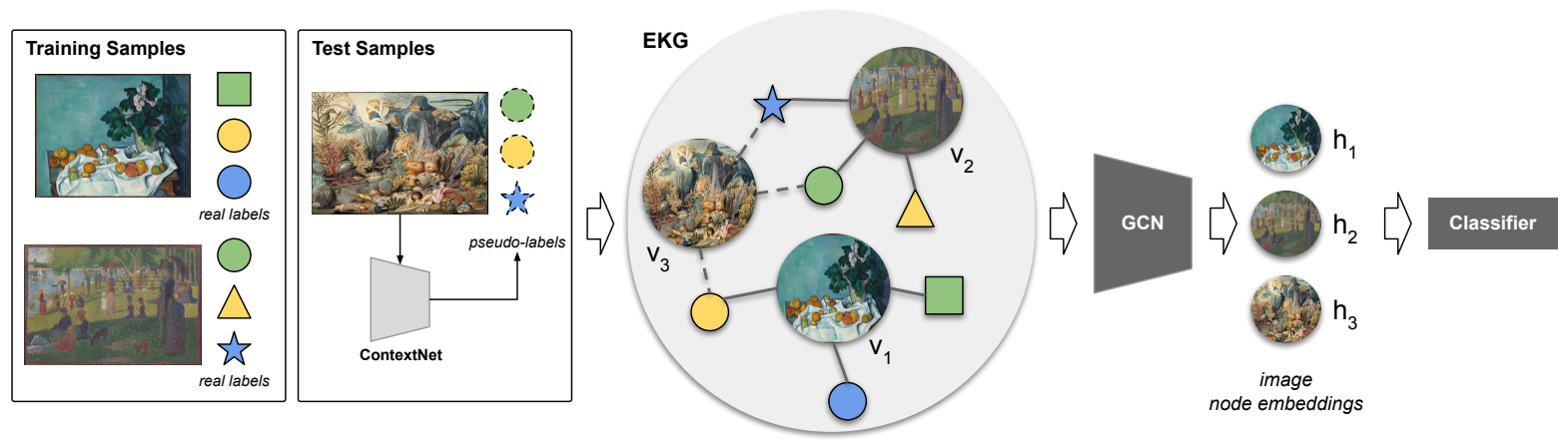

Figure 1: The overview of our proposed framework, named GCNBoost. The input are artworks with their labels (shapes) and unlabeled data that we pseudo-label with a pre-trained (state-of-the-art) model on artwork classification. Artworks and their labels are used to build an EKG that is fed into a GCN and the output embedding is used to build the final classifier. We do not show the initial embeddings of the EKG's nodes that are obtained with ResNet50 [14] for images and node2vec [13] for labels.

labeled data and unlabeled data, facilitating for the same reason classification through labels propagation.

In this paper, we build our EKG based on a given set of entities (images) with their attributes (multiple labels) relying on labeled data as well as unlabeled data, to which we assign pseudo-labels. This EKG also captures relations between the different entities of the dataset. We learn embeddings for all nodes in the graph using a graph convolutional network (GCN). These embeddings are then used for artwork classification to predict multiple labels (i.e. multiple attributes) of a piece of art in a transductive learning framework (see Figure 1 for an overview). The proposed model is evaluated on the SemArt dataset [12], and the Buddha statues dataset [31], for eight different classification tasks, showing significant improvement with respect to previous work.

The main contributions of the paper are summarized as follows:

(1) We propose to build an EKG accounting for both labeled and unlabeled data by using preliminary assigned pseudo-labels.

(2) We devise a framework for digital art analysis that leverages GCNs to compute distinct artwork embeddings, which we show to be robust to unbalanced data.

(3) We evaluate our approach against state-of-the-art methods, obtaining higher accuracy on two different artistic domains: fine-art paintings and Buddha statues.

\section{RELATED WORK}

In this section we limit the discussion to the task of automatic art analysis (Section 2.1), and how it can be benefited from both image classification with GCNs (Section 2.2) and label propagation (Section 2.3).

\subsection{Automatic Artwork Classification}

Historically, the task of automatic art analysis was initially addressed using handcrafted features to describe the visual content of a digitized artwork $[5,16,26]$. Those handcrafted features ranged from color [42] or brushwork [19] detection to scale-invariant local features [32], and were used to classify pieces of art according to their attributes ${ }^{1}$ through SVM classifiers. However, those approaches were bounded by the quality of the features themselves.

With the emergence of machine learning techniques that automatically extract features from an image using pre-trained convolutional neural networks (CNNs), such as ResNet [14] or VGG [34], the need for handcrafted features was replaced. Pre-trained CNNs could capture accurate representations for different kinds of entities, such as text, natural images, or art pieces, and thus, they were extensively used as an off-the-shelf method to classify artworks $[3,11,12,23,24,31]$. CNNs have also been fine-tuned to devise multitask art classifiers [11, 23, 31, 36]. Meanwhile, features extracted from CNNs only contain information about the visual aspect of the image, without considering the cultural and historical context of the artworks. To skirt this issue, the authors of [12] proposed to use a joint visual and textual representation for fine-art paintings, allowing a multimodal analysis and opening the door to study art from the semantic point of view.

To further study art from the semantic perspective, visual information can be complemented with specific knowledge about the art pieces, such as the artists, the period of time, or the style. This allows to incorporate the general context of the artworks, such as the social and historical context, into their representation. For instance, in order to have contextual representations of artworks and their attributes, a multi-task learning approach is developed in [11], allowing different paintings to interact through their attributes. Moreover, KGs can be used to build an accurate representation [11,31] of artworks and their attributes based on KG embedding models such as node2vec [13]. The latter is used in [11,31] jointly with deep visual features, showing state-of-the-art results of painting and Buddha statues classification. Our paper follows the same direction by devising a semantic KG for art analysis. Furthermore, we study how unlabeled data can be used within a transductive setup, in order to improve the quality of artworks' interrelationships in a KGs, facilitating automatic art analysis.

\footnotetext{
${ }^{1}$ e.g. author, style movement, or period of time.
} 

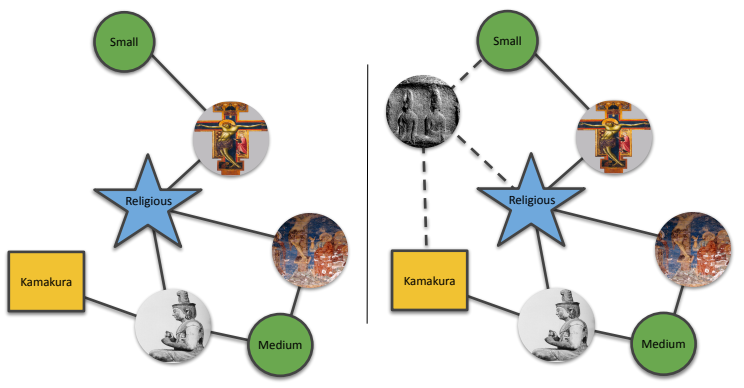

Figure 2: An example of a KG and its EKG. Each node corresponds to either a painting, a Buddha statue or an attribute. The plain lines correspond to existing relationships in the KG (left), while dashed lines are obtained with pseudo-labels to build the EKG (right).

\subsection{Image Classification with GCNs}

In the recent work on image classification, GCNs are receiving more attention thanks to their ability to model the relationships between a set of entities through a KG, which is effective in the tasks of node classification [28, 35] and link prediction [35]. Particularly, GCN are used to model the relations a set of images may have accounting for their labels $[6,22,39,43]$. The basic idea is to combine the classical visual features with GCN embeddings learned over the KG. For example, GCNs are used in [6] to improve multi-labels classification accounting for semantic links between different labels, whereas the authors of [39] improved this idea with WordNet concepts hierarchy, devising a zero-shot classification technique. Finally, the authors in [43] used a weighted adjacency matrix to efficiently model the inter-dependency between image labels. We follow the same idea, introducing pseudo-labeled data, used as true data to train a GCN following a standard label propagation process. However, training GCNs with noisy data is challenging, as they are based on the hypothesis of an isotropic averaging operation, meaning that pseudo-labeled data have the same influence to their neighborhood as the ground truth. To alleviate this issue, we use different pseudo-labels for each artwork.

\subsection{Label propagation}

Label propagation over networks has been a successful strategy to help classification of pseudo-labeled data [29]. It has recently been used for transductive learning with GCNs to incrementally label test data $[8,15]$. The authors in [8] used online pseudo-labeling process for unlabeled data, while the authors in [15] performed the pseudo-labeling offline without changing it at learning time. Our work is a combination of the two. We start with pseudo-labels produced by a pre-trained model, and we refine those pseudo-labels during training.

\section{Approach}

Our task is defined as a multi-label classification problem. Formally, given an artwork $x$, we predict a set of its associated labels $\left\{t_{c} \mid c \in\right.$ $C$ \}, where $t_{c}$ is a label in a certain set $L_{c}$ of labels and $C$ is the set of label category indices. Taking the SemArt dataset [12] as an example, we have Type, School, TimeFrame, and Author in $C$, and each set has specific labels, i.e., portrait in $L_{t y p e}$, Dutch in $L_{\text {school, }}$, and Vincent van $G o g h$ in $L_{\text {author }}$. A straightforward approach to solve this problem is to adopt a multi-task learning framework with a CNNs, in which a single feature extractor is shared among classifiers dedicated for $L_{c}$ 's.

As aforementioned, attributes (or corresponding labels) are rarely independent to each other in a real-world multi-label dataset. A relationship between two labels may be provided as auxiliary knowledge in the dataset or can be extracted from external knowledge sources, such as DBpedia ${ }^{2}$. Such relationships include the time frame of an artwork and its author, stating that the author lived in that period of time (overlapped over the same period of time). For example, the SemArt dataset [12] comes with such auxiliary information that links between labels: Vincent van Gogh is from the Dutch painting school, and all his painting have the same school, which can be represented by an edge connecting author Vincent van Gogh to school Dutch. Such knowledge tells possible correlations among artworks, which can facilitate image representation.

This leads us to reformulating our multi-label classification task with the transductive learning paradigm, explicitly modeling the correlations among labels and artworks in both training and test sets through a KG as in Figure 2. Test images have no labels or missing labels; we thus assign pseudo-labels to make noisy connections in the graph and compute an embedding of each image. This allows to capture different relations between art pieces. That is, when two artworks are from the same author, they are semantically related and connected through a path of length two, and should be closer when compared to non-related artworks (with regards to author nodes).

This transductive learning paradigm, as shown in Figure 1, is core to our classification framework (named GCNBoost): we create an extended knowledge graph (EKG) based on pseudo labels predicted by a model on a training set, then infer multiple artwork classes using a graph convolution network (GCN). The following subsections describe our KG construction, image embedding using the EKG, and training and inference process with pseudo-labels.

\subsection{KG Construction}

A KG is a graph $\mathcal{G}=(\mathcal{V}, \mathcal{E})$, in which the entities and their relations are represented by a collection of nodes $\mathcal{V}$ and edges $\mathcal{E}$, respectively. Nodes in $\mathcal{V}$ are the artworks and their labels (i.e., attributes). The latter can be authors-the entities that created the artworks-or types-categories of art such as portraits or landscapes-of the artworks. The edges in $\mathcal{E}$ represent the relations between two entities in $\mathcal{V}$. The semantic of those relations depend upon the underlying entities. For example, an edge between a certain artwork and a certain author represents the artwork is created by the author. Thus, the KG captures the contextual knowledge and the semantics of the relationships between the artworks and their labels.

Formally, let $\mathcal{X}_{\text {train }}$ and $\mathcal{X}_{\text {test }}$ denote the sets of artworks for training and test, respectively. Artwork $x \in \mathcal{X}_{\text {train }}$ is associated with multiple labels $\left\{t_{c} \mid c \in C\right\}$. The assignment of a label to an artwork gives edge $\left(x, t_{c}\right)$, and the set of all edges are denoted by $\mathcal{W}$. As mentioned above, some auxiliary sources of knowledge can

\footnotetext{
${ }^{2}$ https://wiki.dbpedia.org/
} 
provide explicit relationships among labels $\mathcal{K}=\left\{\left(l_{i}, l_{j}\right) \mid l_{i}, l_{j} \in \mathcal{L}\right\}$, where $\mathcal{L}=\cup_{c} L_{c}$. Using these definitions, sets $\mathcal{V}$ and $\mathcal{E}$ can be given by

$$
\begin{aligned}
& \mathcal{V}=X_{\text {train }} \cup \mathcal{L} \\
& \mathcal{E}=\mathcal{W} \cup \mathcal{K} .
\end{aligned}
$$

For transductive learning, we extend the KG $\mathcal{G}$ with the test set $X_{\text {test }}$. Let $\mathcal{G}^{\prime}=\left(\mathcal{V}^{\prime}, \mathcal{E}^{\prime}\right)$ denote our extended knowledge graph (EKG). $\mathcal{V}^{\prime}$ is a simple extension of $\mathcal{V}$ with the test set, that is,

$$
\mathcal{V}^{\prime}=\mathcal{V} \cup \mathcal{X}_{\text {test }}
$$

Artworks in the test set have no associated labels and so no edges. In order to facilitate the learning of image embeddings, we use an initial guess of labels for the test set, namely pseudo-labels, and add them to the graph. Let $t_{c}^{\prime}=g_{c}(x)$ denote the label in $L_{c}$ predicted by a (separately) trained classifier $g_{c}$ for $x$ in test set $X_{\text {test }}$. We can add this to $\mathcal{E}$ as new nodes and $\left\{\left(x, t_{c}^{\prime}\right)\right\}$ as new edges for all $x \in \mathcal{X}_{\text {test }}$ and all $c \in C$. We thus have our EKG $\mathcal{G}^{\prime}=\left(\mathcal{V}^{\prime}, \mathcal{E}^{\prime}\right)$, where

$$
\mathcal{E}^{\prime}=\mathcal{E} \cup\left\{\left(x, t_{c}^{\prime}\right) \mid x \in \mathcal{X}_{\text {test }}, c \in C\right\} .
$$

\subsection{Image Embedding with EKG}

We adopt a GCN [18] to encode into artwork (and label) embeddings the relationships provided by $\mathcal{G}^{\prime}$. Our GCN has multiple layers, and the $n$-th layer's $(n=1, \ldots, N)$ hidden state $H^{(n)}$ is given using $(n-1)$-th layer's $H^{(n-1)}$ by

$$
H^{(n)}=\operatorname{ReLU}\left(D^{-\frac{1}{2}}(A+I) D^{-\frac{1}{2}} H^{(n-1)} W^{(n)}+b^{(n)}\right) .
$$

$A \in \mathbb{R}^{\left|\mathcal{V}^{\prime}\right| \times\left|\mathcal{V}^{\prime}\right|}$ is the adjacency matrix derived from $\mathcal{G}^{\prime}, D$ is a diagonal matrix of node degrees given by $D_{i i}=\sum_{j} A_{i j}$, and $I$ is the identity. $W^{(n)} \in \mathbb{R}^{d \times d}$ is a matrix of learnable parameters. $H^{(0)} \in \mathbb{R}^{\left|\mathcal{V}^{\prime}\right| \times d}$ is a stack of initial features of nodes, where row vector $h_{i}^{(0)}$ corresponds to the feature vector for node $v_{i}$. For images, we use ResNet50 [14] to obtain the initial feature vector. For labels, initial feature vectors are given by node2vec [13] over $\mathcal{G}^{\prime}$.

With this GCN architecture, we can learn latent relationships between the nodes of the EKG. The adjacency matrix A provides direct relationships among the nodes in $\mathcal{G}^{\prime}$, and the $\mathrm{GCN}$ propagates the information according to the edges. This process is particularly efficient for label nodes that serve as hub-nodes with high degree-as they will be equally important to their neighborhoods and facilitate the task of classification, by short cutting the path between images and labels nodes. For instance, we directly use the relationships between artwork and label nodes, or between label nodes themselves as we can easily interpret such relationships. Meanwhile, the model relies on nodes relationships in general such as indirect relationships through hubs.

\subsection{Training and Inference}

Given the artwork embedding $h_{i}=h_{i}^{(N)}$ for the node $v_{i} \in X_{\text {train }} \cup$ $X_{\text {test }}$, a fully connected (FC) classifier $f_{c}$ with softmax makes prediction for each set $L_{c}$ of labels. Formally,

$$
y_{i c}=f_{c}\left(x_{i}\right)=\operatorname{softmax}\left(W_{c} h_{i}+b_{c}\right),
$$

where $W_{c} \in \mathbb{R}^{\left|L_{c}\right| \times d}$ and $b_{c} \in \mathbb{R}^{\left|L_{c}\right|}$ are learnable parameters. The loss function $\ell$ is given by

$$
\ell=-\sum_{x_{i} \in \mathcal{X}_{\text {train }}} \sum_{c \in C} \sum_{k=1}^{\left|L_{c}\right|} t_{i c k} \log y_{i c k},
$$

where $t_{i c k}$ is the $k$-th element of the one-hot vector of label $t_{i c}$ for $x_{i}$. The loss function is evaluated only over the training set $\mathcal{X}_{\text {train }}$. After training, the $h_{i}$ corresponding to image $x_{i} \in \mathcal{X}_{\text {test }}$ is fed to the $f_{c}$ and softmax to predict its label.

\section{Experiments}

We conducted a thorough experimental evaluation of our framework on both the SemArt and Buddha statues datasets. We showcase performance against state of the art, and robustness with respect to noise in the relationships between nodes of a given graph. After the description of the datasets used in our experiments in Section 4.1, we present our implementation details in Section 4.2. The ablation studies on our approach are gathered in Section 4.3, comparing the importance of using different attributes of art pieces when building the KG as described in Section 3.1. Finally, we compare our classification approach to state-of-the-art competitors, showing significant improvement on the SemArt painting dataset in Section 4.4, and the Buddha statues dataset in Section 4.5.

\subsection{Evaluation Datasets}

To evaluate our artwork classification model, we used two datasets: SemArt [12] and Buddha statues [31].

SemArt dataset The SemArt dataset contains 19,244 train, 1,069 test, and 1,069 validation images of European fine-art paintings. Each painting is labeled with the attributes Author, Title, Date, Technique, Type, School, and TimeFrame. We use the pre-trained ContexNet model [11] to produce the initial pseudo-labels for the test samples. Following previous work [11], we evaluate our models on four classification tasks:

- Type classification (T1.1) consists in classifying each painting in one of the 10 following Types: portrait, landscape, religious, study, genre, still-life, mythological, interior, historical and other.

- School classification (T1.2) aims at assigning a class to each painting from one of the 24 following classes: Italian, Dutch, French, Flemish, German, Spanish, English, Netherlandish, Austrian, Hungarian, American, Danish, Swiss, Russian, Scottish, Greek, Catalan, Bohemian, Swedish, Irish, Norwegian, Polish, Other and Unknown.

- TimeFrame classification (T1.3) targets classification of the painting according to 17 different period of time and one additional class (Unknown) for or TimeFrames that are not considered classes. Each class contains at least 10 training samples.

- Author classification (T1.4) performs painting classification according to 350 different painters. We first do so by including all the authors (although some authors have only one training sample in the dataset). In a second experiment, we filtered authors so that we keep only the ones with at least ten paintings in the training set. 
Buddha statues dataset Similarly, the Buddha statues dataset contains 2,665 samples split into 1,866 train, 533 test, and 266 validation, with the attributes Style, Size, Century of creation, and Dimensions. To compute the pseudo-labels, we trained the proposed neural network (NN) model from [31], which we also use as a baseline. We evaluate our model on the four tasks defined in [31]:

- Statue style classification (T2.1) consists in classifying Buddha faces in one of this four different styles: China, Kamakura period, Nara period, and Heian period.

- Statue size classification (T2.2) assigns a class to each Buddha faces from one of this three different styles: Small, Medium and Big.

- Statue century of creation classification (T2.3) aims at classifying Buddha faces into seven different centuries: V, VI, VII, VIII, IX, XII, XIII. We disregard centuries with few training samples.

- Statue dimensions classification (T2.4) performs Buddha faces classification according to 12 different dimensions ranging from 50 to $1,050 \mathrm{~cm}$.

\subsection{Implementation Details}

At learning time, we use the whole dataset at each iteration, allowing a global optimization without mini-batches. While global training is memory expensive in general, since we have relatively small datasets that fit in memory, it was more convenient and faster than using mini-batches, specially when training on GPUs.

For artwork classification, we train a two-layer GCN as described in Section 3.2 for node classification. Node embeddings are initialized using node2vec for training and validation samples, and randomly for test samples. The dimension of this embedding is 128. The first layer of our GCN takes the initial embedding, and outputs a hidden representation of size 16, following the GCN hyperparameters [18]. This first level GCN embedding is introduced in the second layer which an output size equals to the number of labels $\left|L_{C}\right|$. We train a separate model for each task.

We use Adam [17] for gradient optimization with the learning rate set to 0.001 , and a maximum number of iterations set to 2,000 . We also implement an early stop mechanism with a window size of 100 , i.e. we stop training if the validation loss does not decrease for 100 consecutive iterations.

\subsection{Importance of Pseudo-Labels Assignment}

We first evaluate on the SemArt dataset the effect of assigning pseudo-labels to the test set to build our proposed EKG.

Baselines For comparison, we use two models: the original ContextNet [11], and our proposed model based on EKG but with a random assignment of pseudo-labels ( $S_{0}$ Random initialization).

Pseudo-label assignment In the rest of our models, we initally predict pseudo-labels using the pre-trained ContextNet. To analyse its contribution, we add between one to four categories of pseudolabels at a time, referred as models $S_{1}, S_{2}, S_{3}$, and $S_{\text {all }}$, respectively.

Results are shown in Table 1. Rows 1 and 2 report the accuracies of the baselines, showing that 1) ContextNet [11] is a strong model, and 2) the random initialization does not work at all.
Table 1: Classification results on SemArt. The first part of this table gives the baseline. The following groups give the classification accuracy using one, two, three, then all categories of pseudo-labels to build the KG.

\begin{tabular}{llrrrr}
\hline & & Type & School & TimeFrame & Author \\
& Model & T1.1 & T1.2 & T1.3 & T1.4 \\
\hline 1 & ContextNet [11] & 0.815 & 0.671 & 0.613 & $\mathbf{0 . 6 1 5}$ \\
2 & $S_{0}$ Random intialization & 0.100 & 0.010 & 0.050 & 0.040 \\
\hline 3 & $S_{1}$ Type & 0.807 & 0.368 & 0.136 & 0.013 \\
4 & $S_{1}$ School & 0.296 & 0.718 & 0.154 & 0.035 \\
5 & $S_{1}$ TimeFrame & 0.363 & 0.392 & 0.796 & 0.047 \\
6 & $S_{1}$ Author & 0.352 & 0.420 & 0.271 & 0.181 \\
\hline 7 & $S_{2}$ School_Author & 0.397 & 0.866 & 0.314 & 0.284 \\
8 & $S_{2}$ School_Type & 0.861 & 0.739 & 0.213 & 0.058 \\
9 & $S_{2}$ School_TimeFrame & 0.363 & 0.846 & 0.843 & 0.139 \\
10 & $S_{2}$ Type_Author & 0.899 & 0.486 & 0.295 & 0.302 \\
11 & $S_{2}$ Type_TimeFrame & 0.915 & 0.485 & 0.853 & 0.098 \\
12 & $S_{2}$ Author_TimeFrame & 0.417 & 0.504 & 0.906 & 0.354 \\
\hline 13 & $S_{3}$ School_Author_TimeFrame & 0.461 & 0.882 & $\mathbf{0 . 9 3 3}$ & 0.435 \\
14 & $S_{3}$ Author_TimeFrame_Type & 0.930 & 0.564 & 0.932 & 0.482 \\
15 & $S_{3}$ Author_School_Type & 0.924 & 0.827 & 0.348 & 0.394 \\
16 & $S_{3}$ School_Type_TimeFrame & 0.929 & 0.859 & 0.877 & 0.204 \\
\hline 17 & $S_{\text {all }}$ & $\mathbf{0 . 9 3 9}$ & $\mathbf{0 . 8 8 9}$ & 0.927 & 0.479 \\
\hline
\end{tabular}

Rows 3 to 6 show results when adding only one category of pseudo-labels at a time $\left(S_{1}\right)$. This strategy improves the classification accuracy over the original ContextNet on School by 4 points and TimeFrame by 18 points. Results are close to the baseline on Type. However, we lose 42 points on Author, mostly because of the unbalanced data in this category (further details in Section. 4.4). Moreover, $S_{1}$ strategy only improves accuracy for the categories used to build the graph, obtaining poor results on the others. For example, using School pseudo-labels (Row 4) improves only the results for the same category, School, and obtains poor results on Type, TimeFrame, and Author.

Rows 7 to 12 show results when two categories of pseudo-labels are used to build the EKG $\left(S_{2}\right)$. Results are improved with respect to $S_{1}$ on multiple ways. First, accuracy is enhanced for the considered categories: e.g. using School and Type (Row 8) raises the accuracy for both categories over $S_{1}$ when only using School (Row 4) or Type (Row 3). Second, accuracy is also improved for the other categories, e.g. TimeFrame and Author are better in Row 8 than in Row 3 or Row 4. This gain in accuracy highlights the interest of using existing nodes interrelationships to capture hidden relations between nodes, which leads to improve the accuracy for the classification task. Third, from a qualitative point of view, we observe that the results for some pairs of pseudo-labels are coherent with the way we create the EKG. For example, Type and TimeFrame (Row 11) obtain the best scores among all the $S_{2}$ models because a large number of painting types were famous in a particular period of time, which creates a hub of nodes around TimeFrame, and Type.

Rows 13 to 16 show results combining three categories of pseudolabels $\left(S_{3}\right)$, whereas Row 17 provides accuracies when all the categories are used $\left(S_{\text {all }}\right)$. These strategies further improve results, observing similar conclusions as with strategy $S_{2}$. Meanwhile, $S_{3}$ is better than $S_{\text {all }}$ on TimeFrame. While the difference in accuracy 
Table 2: Comparison of different classification results on SemArt. The row GCNBoost $S_{1}$ to $S_{\text {all }}$ gives different configurations of our classification approach

\begin{tabular}{lrrrr}
\hline & Type & School & TimeFrame & Author \\
Model & T1.1 & T1.2 & T1.3 & T1.4 \\
\hline VGG16 pre-trained [11] & 0.706 & 0.502 & 0.418 & 0.482 \\
ResNet50 pre-trained [11] & 0.726 & 0.557 & 0.456 & 0.500 \\
ResNet152 pre-trained [11] & 0.740 & 0.540 & 0.454 & 0.489 \\
VGG16 fine-tuned [11] & 0.768 & 0.616 & 0.559 & 0.520 \\
ResNet50 fine-tuned [11] & 0.765 & 0.655 & 0.604 & 0.515 \\
ResNet152 fine-tuned [11] & 0.790 & 0.653 & 0.598 & 0.573 \\
ResNet50+Attributes [11] & 0.785 & 0.667 & 0.599 & 0.561 \\
ResNet50+Captions [11] & 0.799 & 0.649 & 0.598 & 0.607 \\
\hline ContextNet MTL [11] & 0.791 & 0.691 & 0.632 & 0.603 \\
ContextNet KGM [11] & 0.815 & 0.671 & 0.613 & 0.615 \\
\hline GCNBoost $S_{1}$ & 0.807 & 0.718 & 0.796 & 0.181 \\
GCNBoost $S_{2}$ & 0.915 & 0.866 & 0.906 & 0.354 \\
GCNBoost $S_{3}$ & 0.930 & 0.882 & $\mathbf{0 . 9 3 3}$ & 0.482 \\
GCNBoost $S_{\text {all }}$ & $\mathbf{0 . 9 3 9}$ & $\mathbf{0 . 8 8 9}$ & 0.927 & 0.479 \\
\hline GCNBoost $S_{\text {all }}{ }^{*}$ (Author filter) & - & - & - & $\mathbf{0 . 7 0 2}$ \\
\hline
\end{tabular}

is only one point, this suggests that adding extra information to the EKG may incorporate too much noise. Thus, adding three categories of pseudo-labels seems to always improve the accuracy for the SemArt dataset, while four categories may or may not improve the results.

\subsection{SemArt Evaluation}

Next, we compare our approach to a series of methods that reported state-of-the-art results on the SemArt dataset, namely:

- Pre-trained Networks [11]. VGG16 [34], ResNet50 [14], and ResNet152 [14] trained for natural image classification.

- Fine-tuned Networks [11]. VGG16, ResNet50, and ResNet152 fine-tuned on the SemArt dataset.

- ResNet50+Attributes [11]. Combination of ResNet50 features with the predicted attributes by one of the three previous fine-tuned models.

- ResNet50+Captions [11]. Combination of ResNet50 features with a generated caption embeddings [41].

- ContextNet models [11]. ContextNet includes a multi-task learning model (ContextNet MTL), which learns the classification of the four categories of a painting as a four shared tasks, and a knowledge graph model (ContextNet KGM), which is the one used in this work to predict the pseudolabels. ContextNet KGM builds and uses an artistic KG to model relation between nodes and to perform classification, combining features extracted with a ResNet50 [14] for visual features and the model node2vec [13] for nodes embedding.

The models above can be divided into two groups: 1) local, disregarding the context (Pre-trained Networks, Fine-tuned Networks, and ResNet50+Captions); and 2) global, incorporating as much context as possible (ResNet50+Attributes, ContextNet). The scores for
Table 3: Classification results on Buddha dataset. $S_{\text {all }}$ is using style, size, century, and dimensions to create the EKG

\begin{tabular}{llrrrr}
\hline & & Style & Size & Century & Dimensions \\
& Model & T2.1 & T2.2 & T2.3 & T2.4 \\
\hline \multirow{2}{*}{1} & NN (original) $[31]$ & $\mathbf{0 . 9 8}$ & 0.78 & 0.78 & 0.78 \\
2 & NN (retrained) & 0.58 & 0.65 & 0.76 & 0.46 \\
\hline \multirow{2}{*}{3} & $S_{0}$ Random intialization & 0.23 & 0.30 & 0.13 & 0.08 \\
4 & GCNBoost $S_{1}$ & 0.57 & 0.68 & 0.74 & 0.47 \\
5 & GCNBoost $S_{2}$ & 0.59 & 0.85 & 0.80 & 0.76 \\
6 & GCNBoost $S_{3}$ & 0.88 & 0.86 & 0.86 & 0.84 \\
7 & GCNBoost $S_{\text {all }}$ & 0.92 & $\mathbf{0 . 9 4}$ & $\mathbf{0 . 9 3}$ & $\mathbf{0 . 9 0}$ \\
\hline
\end{tabular}

the different methods are obtained from [11], except for ContextNet that are computed using the pre-trained models on Github. ${ }^{3}$

Results are reported in Table 2. The proposed GCN classification strategy $\left(S_{\text {all }}\right)$ outperforms previous methods by a large margin in all the categories except Author, where the accuracy is very low compared to ContextNet. One can note that ContextNet MTL and ContextNet KGM are complementary on the four categories of attributes, as they model the global nodes interrelationships differently, but they also obtain low scores on TimeFrame and Author. This can be explained by the imbalance in the number of training samples in those categories (especially for Author). While our GCNBoost model is able to recover the problem for Type, School and TimeFrame (they have a only small unbalancing), the same approach failed with Author.

To understand the imbalance problem for the Author category, in Figure 3, we plot the degrees distribution of the EKG nodes for the different attributes (one sub-plot per attribute). We can see that all the attributes are imbalanced. However, the attributes in Author (Figure 4d) present a huge imbalance: there are around 2, 000 Author nodes with small connectivity (small number of associated paintings) and less than 200 nodes with medium to high connectivity (more than 50 associated paintings). To show how this data imbalance affects the result in our proposed model, we disregard Author nodes with a degree less than five (i.e. to have at least 10 training examples). The result for this setup is given as GCN $S_{\text {all }}{ }^{*}$ (Author filter) (bottom line of Table 2). We can see that by removing painting data points with few learning samples, we are able to improve the accuracy. However, the last result is not directly comparable with the rest of the approaches in Table 2 since we trimmed the training set to have enough training samples.

\subsection{Buddha Statues Evaluation}

We apply our approach on the Buddha statues dataset following the same process as for the SemArt dataset. We use as a baseline the NN classifier defined in the original work [31]. It is a fully connected layer followed by a softmax activation with categorical cross entropy loss based on image features (the best results in the paper are given with ResNet-50 features [31]). This model is used in two setups:

- NN original is exactly the same architecture and setup as given by the authors of the original paper [31]. It is trained on

\footnotetext{
${ }^{3}$ https://github.com/noagarcia/context-art-classification
} 


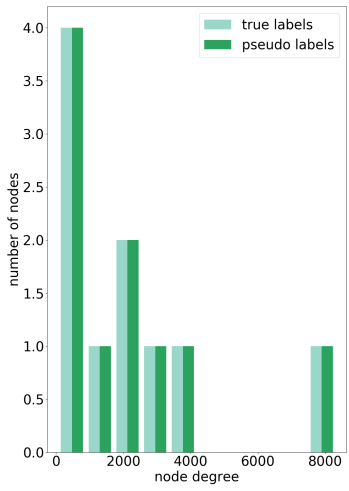

(a) Type

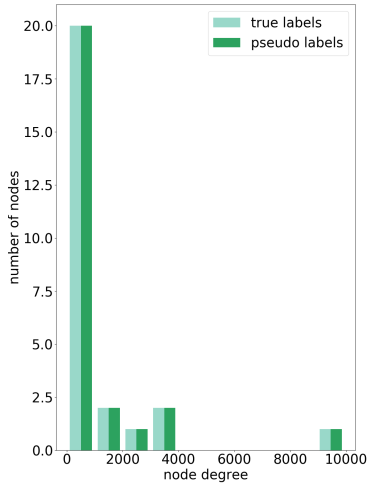

(b) School

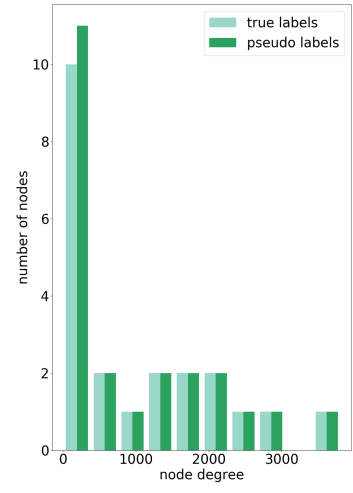

(c) TimeFrame

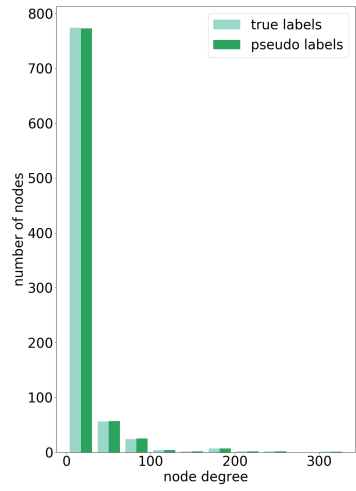

(d) Author

Figure 3: Distribution of nodes degrees for the four categories (Type, School, TimeFrame, Author) based on the true classes for test and train data, and the pseudo-labels for the test.

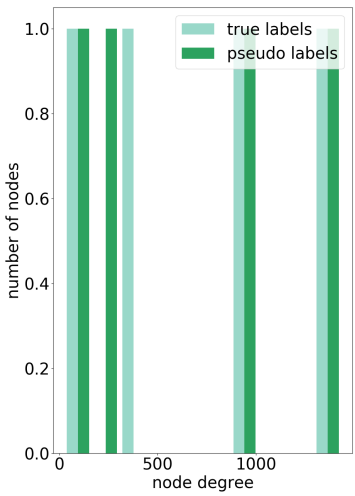

(a) Style

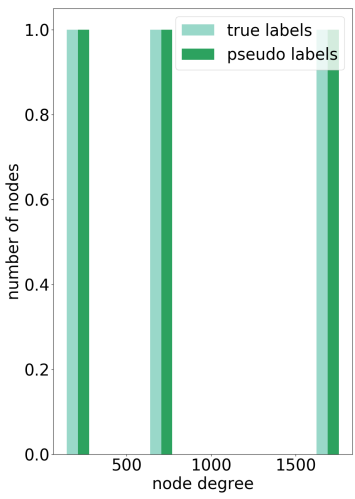

(b) Size

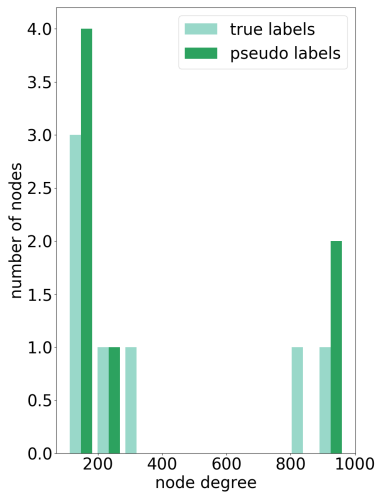

(c) Century

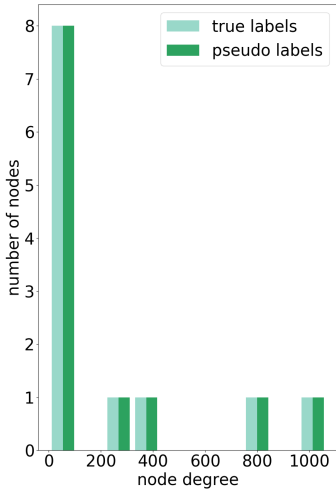

(d) Dimensions

Figure 4: Distribution of nodes degrees for the four categories (Style, Size, Century, Dimensions) based on the true classes for test and train data, and the pseudo-labels for the test.

a set of 3,334 images; however some images do not have all of the four different attributes (sparse data). This setup uses a $70 \%-30 \%$ split of the dataset with 5 -fold cross validation, and does not keep sample for a test set.

- NN retrained is also the same architecture as given by the authors of the original paper [31], but with a different setup, that is common to all other experiments for Buddha statues. We consider a $70 \%-20 \%$ split of the Buddha statues dataset for train and validation, and keep the $10 \%$ of the remaining statues as an independent test set. Furthermore, each statue we consider bears all of the four attributes of our tasks, with a total of 2,665 images.

We build the EKG in the same spirit as for SemArt, but of course using different attributes. We leverage on the $\mathrm{NN}$ model (retrained) to build the pseudo-labels. We show in Figure 4 the node distributions for this EKG, and notice some imbalance (although less than for the SemArt dataset). Our GCNBoost shows robustness to this issue, by mitigating the different attributes of a statue as we discussed in the previous section.

To further investigate the impact of each attribute, we use the same strategy of adding one at a time. The results are gathered in Table 3. The first row gives the best results reported for the NN of the original paper [31]. The second row gives our baseline with the retrained $\mathrm{NN}$ on our experimental setup, while the following rows give our GCNBoost model for each different strategy. The results of the NN models greatly vary across the different attributes, even in the best reported case. In contrast, GCNBoost is able to improve most of the classification results, in spite of the limited available 

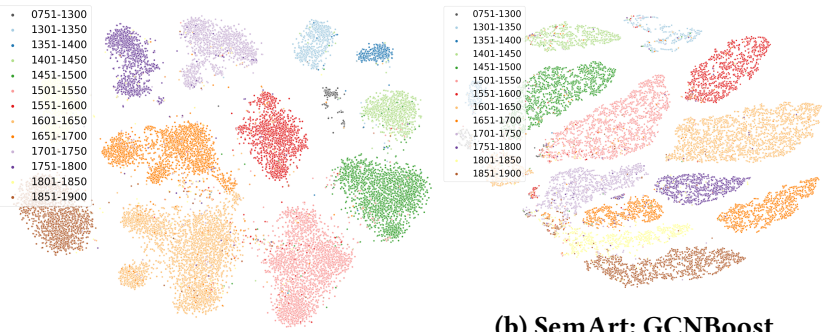

(a) SemArt: ContexNet

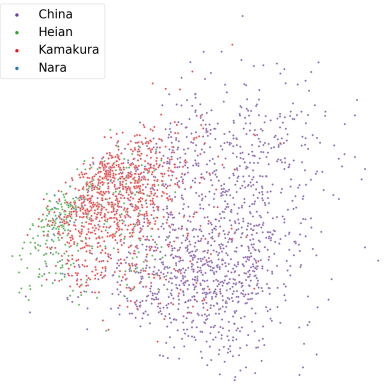

(c) Buddha: NN (retrained)

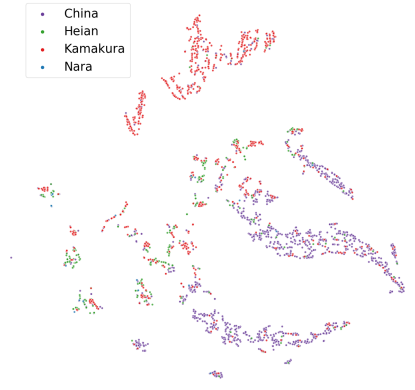

(d) Buddha: GCNBoost

Figure 5: Visualization using tSNE [37] of the embeddings of ContextNet [11] (a) and our GCNBoost model (b), learned when predicting the category TimeFrame on SemArt [12], and the NN [31] (c) and our GCNBoost (d) on Buddha statues [31].

data (only 2,665 samples). Moreover, we also notice a consistent accuracy increase from the worst model, $S_{0}$ with random pseudolabels assignment, to the best model, $S_{\text {all }}$ that includes all artwork relationships.

Finally, the original reported NN results still show a better accuracy on Style classification (T2.1). This may be explained by the fact that it uses a 5-fold cross validation, with more training data as compared to our protocol. GCNBoost and the retrained NN baseline retain $10 \%$ of artworks to form a separated test dataset (that was not done in the original paper). This difference of setup might explain the difference between performances of the two experiments.

\section{Discussion}

In order to understand the quality of our approach with the EKG and GCN, we further investigate the role of node degree distributions in the EKG, as well as the ability of GCNBoost to cluster the different art pieces while sharing similar attributes just as homophily in real world networks.

Homophily [4] is a widely studied concept in complex networks. It follows the intuition that the more entities share attributes, the more they tend to assemble together (for example, people in social ties [25]), bringing cohesion to clusters of entities [30]. Combining visual features and the KG builds upon this idea: visually similar paintings have a high chance to share some semantic properties. While the generation of pseudo-labels introduce some level of noise, the GCN mitigates this noise by considering neighborhood at a larger level, comparable to a smoothing process. This effect however

comes to a limitation when information is insufficient. This is due to the long-tail distribution of node degrees, typical of real-world data [7]: a few observations tend to be extremely well connected, while many observations are little to not connected at all. As a consequence, we can observe a very imbalanced distribution of labels, and the Author class makes one good example (see Figure 4d). It thus becomes difficult, from the graph perspective, for a random walk to reach authors with a low degree (i.e. a low number of paintings), and then predict them to any given painting. Investigating different loss functions (such as focal loss [20] or distribution-balanced loss [40]) may help mitigate this issue.

We further visualize using tSNE [37] the embeddings of ContexNet (Figure 5a) and GCNBoost (Figure 5b) learned when predicting the category TimeFrame on SemArt, as well as the embeddings of the baseline NN model (Figure 5c) and GCNBoost (Figure 5d) when predicting the category Style on Buddha dataset. Following the idea of homophily [4] we can see that ContexNet (Figure 5) globally clusters well the different timeframe categories but still displays some noise in between the clusters. The Resnet50 based $\mathrm{NN}$ model puts everything in the same cluster (Figure 5c). In contrast, GCNBoost (Figure 5b and Figure $5 \mathrm{~d}$ ) tends to display better separated clusters (but not only limited to separating artworks based on their timeframe or their style). We keep for future work the investigations that might reveal the combinations of visual and semantic attributes explaining the clusters.

\section{Conclusion and Perspectives}

This paper shows a method that increases the effectiveness of the use of a knowledge graph (KG) in the context of multi-label classification with a transductive learning framework. Our method leverages on the inclusion of unlabelled data, which get pseudo labels attributed through label propagation enriching the KG, forming an extended KG (EKG). Using graph convolution networks (GCN) on the EKG, we have shown experimentally improvement on different classification tasks of two different artwork datasets (SemArt [12] and Buddha statues [31]). Experiments have additionally shown that our method can help mitigate some level of imbalance in the data. To further address this issue of imbalanced data, we further plan to study different strategies for pseudo-label assignment (such as incremental assignment) and the use of different loss functions in the GCN (such as the focal loss [21]).

These results open perspectives for tapping into the richness that unlabeled data can provide, and improve automatic art analysis. This should guide our future work towards zero-shot learning for automatic art analysis.

\section{Acknowledgments}

This work is partly supported by JSPS KAKENHI Grant Numbers JP18H03571, JP18H03264, and JP20K19822. 


\section{References}

[1] Marilena Alivizatou-Barakou, Alexandros Kitsikidis, Filareti Tsalakanidou, Kosmas Dimitropoulos, Chantas Giannis, Spiros Nikolopoulos, Samer Al Kork, Bruce Denby, Lise Buchman, Martine Adda-Decker, et al. 2017. Intangible cultural heritage and new technologies: challenges and opportunities for cultural preservation and development. In Mixed reality and gamification for cultural heritage. Springer, 129-158.

[2] Kazuki Ashihara, Cheikh Brahim El Vaigh, Chenhui Chu, Benjamin Renoust, Noriko Okubo, Noriko Takemura, Yuta Nakashima, and Hajime Nagahara. 2020 Improving topic modeling through homophily for legal documents. Applied Network Science 5, 1 (2020), 1-20.

[3] Yaniv Bar, Noga Levy, and Lior Wolf. 2014. Classification of artistic styles using binarized features derived from a deep neural network. In European conference on computer vision. Springer, 71-84.

[4] Stephen P Borgatti, Ajay Mehra, Daniel J Brass, and Giuseppe Labianca. 2009. Network analysis in the social sciences. science 323, 5916 (2009), 892-895.

[5] Gustavo Carneiro, Nuno Pinho Da Silva, Alessio Del Bue, and João Paulo Costeira 2012. Artistic image classification: An analysis on the printart database. In European conference on computer vision. Springer, 143-157.

[6] Zhao-Min Chen, Xiu-Shen Wei, Peng Wang, and Yanwen Guo. 2019. Multi-label image recognition with graph convolutional networks. In Proceedings of the IEEE/CVF Conference on Computer Vision and Pattern Recognition. 5177-5186.

[7] Aaron Clauset, Cosma Rohilla Shalizi, and Mark EJ Newman. 2009. Power-law distributions in empirical data. SIAM review 51, 4 (2009), 661-703.

[8] Matthijs Douze, Arthur Szlam, Bharath Hariharan, and Hervé Jégou. 2018. Lowshot learning with large-scale diffusion. In Proceedings of the IEEE Conference on Computer Vision and Pattern Recognition. 3349-3358.

[9] Cheikh Brahim El Vaigh, Guillaume Le Noé-Bienvenu, Guillaume Gravier, and Pascale Sébillot. 2020. IRISA System for Entity Detection and Linking at CLEF HIPE 2020. In CEUR Workshop Proceedings.

[10] Cheikh Brahim El Vaigh, François Torregrossa, Robin Allesiardo, Guillaume Gravier, and Pascale Sébillot. 2020. A correlation-based entity embedding approach for robust entity linking. In 2020 IEEE 32nd International Conference on Tools with Artificial Intelligence (ICTAI). IEEE, 949-954.

[11] Noa Garcia, Benjamin Renoust, and Yuta Nakashima. 2019. Context-aware embeddings for automatic art analysis. In Proceedings of the International Conference on Multimedia Retrieval. 25-33.

[12] Noa Garcia and George Vogiatzis. 2018. How to read paintings: semantic art understanding with multi-modal retrieval. In Proceedings of the European Conference on Computer Vision (ECCV). 0-0.

[13] Aditya Grover and Jure Leskovec. 2016. node2vec: Scalable feature learning for networks. In Proceedings of the International Conference on Knowledge Discovery and Data Mining. 855-864.

[14] Kaiming He, Xiangyu Zhang, Shaoqing Ren, and Jian Sun. 2016. Deep residual learning for image recognition. In Proceedings of the IEEE conference on computer vision and pattern recognition. $770-778$.

[15] Ahmet Iscen, Giorgos Tolias, Yannis Avrithis, and Ondrej Chum. 2019. Label Propagation for Deep Semi-Supervised Learning. In Conference on Computer Vision and Pattern Recognition (CVPR).

[16] Fahad Shahbaz Khan, Shida Beigpour, Joost Van de Weijer, and Michael Felsberg. 2014. Painting-91: a large scale database for computational painting categorization. Machine vision and applications 25, 6 (2014), 1385-1397.

[17] Diederik P. Kingma and Jimmy Ba. 2015. Adam: A Method for Stochastic Optimization. In Proceedings of the International Conference on Learning Representations.

[18] Thomas N. Kipf and Max Welling. 2017. Semi-Supervised Classification with Graph Convolutional Networks. In 5th International Conference on Learning Representations, ICLR 2017, Toulon, France, April 24-26, 2017, Conference Track Proceedings. https://openreview.net/forum?id=SJU4ayYgl

[19] Jia Li, Lei Yao, Ella Hendriks, and James Z Wang. 2011. Rhythmic brushstrokes distinguish van Gogh from his contemporaries: findings via automated brushstroke extraction. IEEE transactions on pattern analysis and machine intelligence 34, 6 (2011), 1159-1176.

[20] Tsung-Yi Lin, Priya Goyal, Ross Girshick, Kaiming He, and Piotr Dollár. 2017. Focal loss for dense object detection. In Proceedings of the IEEE international conference on computer vision. 2980-2988.

[21] Tsung-Yi Lin, Priya Goyal, Ross Girshick, Kaiming He, and Piotr Dollár. 2017 Focal loss for dense object detection. In Proceedings of the IEEE international conference on computer vision. 2980-2988.

[22] Zheng Liu, Zidong Jiang, Wei Feng, and Hui Feng. 2020. OD-GCN: Object Detection Boosted by Knowledge GCN. In 2020 IEEE International Conference on Multimedia \& Expo Workshops (ICMEW). IEEE, 1-6.

[23] Daiqian Ma, Feng Gao, Yan Bai, Yihang Lou, Shiqi Wang, Tiejun Huang, and LingYu Duan. 2017. From part to whole: who is behind the painting? In Proceedings of the 25th ACM international conference on Multimedia. 1174-1182.

[24] Hui Mao, Ming Cheung, and James She. 2017. Deepart: Learning joint representations of visual arts. In Proceedings of the 25th ACM international conference on Multimedia. 1183-1191.
[25] Miller McPherson, Lynn Smith-Lovin, and James M Cook. 2001. Birds of a feather: Homophily in social networks. Annual review of sociology 27, 1 (2001), 415-444.

[26] Thomas Mensink and Jan Van Gemert. 2014. The rijksmuseum challenge: Museum-centered visual recognition. In Proceedings of International Conference on Multimedia Retrieval. 451-454.

[27] Foster Provost and Venkateswarlu Kolluri. 1999. A survey of methods for scaling up inductive algorithms. Data mining and knowledge discovery 3, 2 (1999), 131169

[28] Mohammad Rasool Izadi, Yihao Fang, Robert Stevenson, and Lizhen Lin. 2020. Optimization of Graph Neural Networks with Natural Gradient Descent. arXiv e-prints (2020), arXiv-2008.

[29] Benjamin Renoust, Gene Cheung, and Shin'Ichi Satoh. 2017. Estimating political leanings from mass media via graph-signal restoration with negative edges. In 2017 IEEE International Conference on Multimedia and Expo (ICME). IEEE, 10091014.

[30] Benjamin Renoust, Guy Melançon, and Marie-Luce Viaud. 2014. Entanglement in multiplex networks: understanding group cohesion in homophily networks. In Social Network Analysis-Community Detection and Evolution. Springer, 89-117.

[31] Benjamin Renoust, Matheus Oliveira Franca, Jacob Chan, Noa Garcia, Van Le, Ayaka Uesaka, Yuta Nakashima, Hajime Nagahara, Jueren Wang, and Yutaka Fujioka. 2019. Historical and modern features for Buddha statue classification. In Workshop on Structuring and Understanding of Multimedia heritAge Contents. 23-30.

[32] Lior Shamir, Tomasz Macura, Nikita Orlov, D Mark Eckley, and Ilya G Goldberg. 2010. Impressionism, expressionism, surrealism: Automated recognition of painters and schools of art. ACM Transactions on Applied Perception 7, 2 (2010), $1-17$.

[33] Weiwei Shi, Yihong Gong, Chris Ding, Zhiheng MaXiaoyu Tao, and Nanning Zheng. 2018. Transductive semi-supervised deep learning using min-max features. In Proceedings of the European Conference on Computer Vision (ECCV). 299-315.

[34] Karen Simonyan and Andrew Zisserman. 2015. Very Deep Convolutional Networks for Large-Scale Image Recognition. In Proceedings of International Conference on Learning Representations, Yoshua Bengio and Yann LeCun (Eds.)

[35] Devendra Singh Dhami, Siwen Yan, and Sriraam Natarajan. 2021. Bridging Graph Neural Networks and Statistical Relational Learning: Relational One-Class GCN. arXiv e-prints (2021), arXiv-2102.

[36] Gjorgji Strezoski and Marcel Worring. 2018. Omniart: a large-scale artistic benchmark. ACM Transactions on Multimedia Computing, Communications, and Applications (TOMM) 14, 4 (2018), 1-21.

[37] Laurens Van der Maaten and Geoffrey Hinton. 2008. Visualizing data using t-SNE. Journal of machine learning research 9, 11 (2008).

[38] Xin Wang and Weixue Chen. 2020. Knowledge Graph Data Management: Models, Methods, and Systems. In International Conference on Web Information Systems Engineering. Springer, 3-12.

[39] Xiaolong Wang, Yufei Ye, and Abhinav Gupta. 2018. Zero-shot recognition via semantic embeddings and knowledge graphs. In Proceedings of the IEEE conference on computer vision and pattern recognition. 6857-6866.

[40] Tong Wu, Qingqiu Huang, Ziwei Liu, Yu Wang, and Dahua Lin. 2020. Distributionbalanced loss for multi-label classification in long-tailed datasets. In European Conference on Computer Vision. Springer, 162-178.

[41] Kelvin Xu, Jimmy Ba, Ryan Kiros, Kyunghyun Cho, Aaron Courville, Ruslan Salakhudinov, Rich Zemel, and Yoshua Bengio. 2015. Show, attend and tell: Neural image caption generation with visual attention. In International conference on machine learning. 2048-2057.

[42] Marchenko Yelizaveta, Chua Tat-Seng, and Aristarkhova Irina. 2005. Analysis and retrieval of paintings using artistic color concepts. In 2005 IEEE International Conference on Multimedia and Expo. IEEE, 1246-1249.

[43] Dehai Zhang, Menglong Cui, Yun Yang, Po Yang, Cheng Xie, Di Liu, Beibei Yu, and Zhibo Chen. 2019. Knowledge graph-based image classification refinement. IEEE Access 7 (2019), 57678-57690.

[44] Dengyong Zhou, Olivier Bousquet, Thomas Navin Lal, Jason Weston, and Bernhard Schölkopf. 2003. Learning with Local and Global Consistency. In International Conference on Neural Information Processing Systems (Whistler, British Columbia, Canada) (NIPS’03). 321-328. 